\title{
Feeding relationships in Mediterranean bathyal assemblages elucidated by stable nitrogen and carbon isotope data
}

\author{
N. V. C. Polunin ${ }^{1, *}$, B. Morales-Nin ${ }^{2}$, W. E. Pawsey ${ }^{1}$, J. E. Cartes ${ }^{3}$, J. K. Pinnegar ${ }^{1}$, J. Moranta ${ }^{2}$ \\ ${ }^{1}$ Department of Marine Sciences \& Coastal Management, University of Newcastle, Newcastle upon Tyne NE1 7RU, \\ United Kingdom \\ ${ }^{2}$ Consejo Superior de Investigaciones Cientificas-Universitat de les Illes Balears, Institut Mediterrani d'Estudis Avançats, \\ Miguel Marqes 21, 07190 Esporles, Mallorca, Spain \\ ${ }^{3}$ Consejo Superior de Investigaciones Cientificas, Instituto de Ciencias del Mar, Paseo Juan de Borbón s/n, \\ 08039 Barcelona, Spain
}

\begin{abstract}
Fishes and invertebrates were collected from the continental slope southwest of the Balearic Islands, Mediterranean, in October 1996 and May 1998 from 3 faunally-distinct depth zones: 200 to $800 \mathrm{~m}$ (upper slope), 800 to $1425 \mathrm{~m}$ (middle slope) and 1425 to $1800 \mathrm{~m}$ (lower slope). Differences in $\delta^{15} \mathrm{~N}$ and $\delta^{13} \mathrm{C}$ between October 1996 and May 1998 were observed only in the crustaceans Aristeus antennatus and Nematocarcinus exilis. There were few differences among depths in $\delta^{15} \mathrm{~N}$ or $\delta^{13} \mathrm{C}$ of benthic fishes or crustaceans: the shrimps $A$. antennatus and Acanthephyra eximia showed higher $\delta^{15} \mathrm{~N}$ values at greater depth, and $\delta^{13} \mathrm{C}$ values of the fishes Alepocephalus rostratus (Alepocephalidae) and Coelorhynchus coelorhynchus (Macrouridae) also tended to increase with increasing depth. Differences in plankton and suprabenthos were also observed among zones (at depth, greater $\delta^{15} \mathrm{~N}$ values of gelatinous plankton, calanoid copepods and peracarids were recorded, along with smaller $\delta^{13} \mathrm{C}$ values of gelatinous plankton and peracarids). These are attributed to a microbially-mediated increase in $\delta^{15} \mathrm{~N}$ and a reduction in $\delta^{13} \mathrm{C}$ of the particulate material remaining after preferential utilization of labile organic matter in the stable and relatively high $\left(13^{\circ} \mathrm{C}\right)$ temperature of the Mediterranean water column. Strong positive correlations between $\delta^{15} \mathrm{~N}$ and $\delta^{13} \mathrm{C}$ data for plankton, fishes and crustaceans indicated a single primary source material for these communities, which is attributed to marine snow.
\end{abstract}

KEY WORDS: Deep-sea $\cdot$ Mesozooplankton $\cdot$ Fishes $\cdot$ Crustaceans $\cdot$ Suprabenthos $\cdot$ Mediterranean $\cdot$ Bathyal

\section{INTRODUCTION}

Studies of the feeding relationships of deep-sea organisms are very limited and have concentrated primarily on gut-content data, and these data have substantial limitations. For example, gut contents reflect diets at particular points in time and space, and severely neglect certain types of dietary materials such as gelatinous plankton and detritus that may nevertheless be very important in the sustenance of marine

*E-mail: n.polunin@ncl.ac.uk food-webs. Other limitations include problems associated with the voiding of gut contents upon capture, and the very sporadic feeding of many carnivorous species. Furthermore, many animals crush or grind their food, such that identification of prey becomes very difficult, and it is not often clear which components of diets are actually assimilated, particularly when refractory or amorphous foods are ingested (Pinnegar \& Polunin 2000). Such difficulties are especially great in the study of food webs in the deep ocean.

The use of stable nitrogen and carbon isotopes for the study of trophic interactions is now common in aquatic ecosystems (e.g. Pinnegar \& Polunin 2000). 
Stable isotopes can provide an indication of the prey assimilated over a considerable period of time (Hesslein et al. 1993) and elucidate aspects of both the origin and transformation of organic matter ingested. Stable nitrogen isotopes have been used to help define the trophic level of organisms (Vander Zanden et al. 1997). Carbon isotopes may be useful indicators of sources of production (e.g. Harrigan et al. 1989). Given the constraints and paucity of gut-content data, stable isotopes can clearly help to elucidate some aspects of the structure of deep-sea food webs.

Inputs of material to the deep ocean include falls from large animals such as sharks (e.g. Witte 1999) and export of material from littoral plants such as macroalgae (Vetter \& Dayton 1999). However, the main input of primary production into this environment is considered to be the downward flux from the epipelagic zone of aggregated detrital material (e.g. faecal pellets and plankton exoskeletons) and zooplankton (e.g. Miquel et al. 1994) which constitute what is loosely referred to as 'marine snow' (e.g. Sumich 1999). The flux of this material may be rapid (Riemann 1989), and phytodetritus can at times be up to $1 \mathrm{~cm}$ thick on the sea floor (Smith et al. 1996). With increasing depth, the flux tends to decrease (Miquel et al. 1994, Sumich 1999) and change in character (Williams \& Gordon 1970); it also tends to vary temporally in response to changes in production (Riemann 1989, Miquel et al. 1994). The supply of food is generally considered as the main limiting factor for most deep-sea communities (Gage \& Tyler 1991), and consequently marine snow constitutes a valuable food resource for deep-sea microbes, metazoans and bathyal detritivores (Smith et al. 1996). Bacteria may have important ecological roles in the highly oligotrophic deep-Mediterranean environments, for example through decomposition of particulate matter derived from the upper layers (Danovaro et al. 1993). In spite of the evident role of marine snow in food supply, there has been little attempt to assess the diversity of source materials consumed by organisms in the Mediterranean bathyal.

Relative to the Atlantic coast of western Europe, the Mediterranean bathyal assemblage (1500 to $2500 \mathrm{~m}$ ) is impoverished in numbers of species (although exhibiting some endemism; e.g. Moranta et al. 1998). Overall abundance is also well below that of Atlantic coasts (Pérès 1985), and generally declines with depth (e.g. Cartes 1998). The Mediterranean deep sea constitutes a distinct environment, with a high and almost constant temperature (around $13^{\circ} \mathrm{C}$ ) and salinity (Fredj \& Laubier 1985) below $200 \mathrm{~m}$. It has a characteristically low concentration of oxygen (Randall \& Farrell 1997) and there is often a scarcity of food available to the benthos (Pérès 1985). In the western Mediterranean, picoplankton $(>2 \mu \mathrm{m})$ is responsible for up to $50 \%$ of the primary production; this is exploited by the microbial food web (Azam et al. 1983) and may only inefficiently support the requirements of fishes (Randall \& Farrell 1997). Waters around the SW Balearic Islands are as a rule particularly oligotrophicic (e.g. Emelyanov 1972); however phytoplankton blooms do sometimes occur, and winter and summer peaks in primary production have been recorded (Estrada et al. 1985). Temporal changes in the food available to bathyal communities are therefore to be expected and may be detectable in isotopic data.

Community structure changes markedly as a function of depth (Stefanescu et al. 1993), and within the region adjacent to the SW Balearic Islands there are 3 different fish assemblages; an upper slope assemblage (US) from 200 to $800 \mathrm{~m}$ depth, a middle slope assemblage (MS) from 800 to $1425 \mathrm{~m}$ and a lower slope assemblage (LS) below 1425 m (Moranta et al. 1998). The MS is characterized by higher biomass, abundance and mean fish weight than the LS (Moranta et al. 1998), and similar depth zones exist for assemblages of decapod crustaceans (Maynou \& Cartes 2001). In the neighbouring Catalan Sea, the LS is characterized by smaller, more sedentary species with lower energy requirements (Stefanescu et al. 1993, Cartes 1998). Vertical migrations occur, but movements and distributions tend to be complex and their implications for vertical material fluxes from the epipelagic are unknown (Andersen et al. 1992, Macquart-Moulin 1993); an important question is whether, in spite of such migrations, trophodynamic differences occur among communities at different depths.

Among deep-sea fishes are benthic species which ambush prey, forage slowly on the sediment surface, or scavenge large carcasses falling from above, and benthopelagic species, which possess buoyancy mechanisms and enter the water column to feed (Mahaut et al. 1990, Koslow 1996). The scavengers have been observed by the use of baited cameras, but very little is known about them.

The main aim of the present study was to explore the pathways potentially supporting Mediterranean bathyal slope communities. The specific hypotheses were that (1) food-web structure differs between depth zones, (2) deep-sea food webs respond to seasonal changes in the photic zone, and (3) there is only 1 main food source in the Mediterranean deep-sea, i.e. marine snow. We sought to test these hypotheses by analyses of the stable isotopes of carbon and nitrogen.

\section{METHODS}

Sample collection was carried out from the EV 'Garcia del Cid' in October 1996 and May 1998 off the 
Balearic Islands (Algerian Basin, western Mediterranean) south of the Ibiza and Formentera islands (Fig. 1). Samples of large fishes and decapod crustaceans were obtained by a semi-balloon otter trawl towed at a speed of $5 \mathrm{~km} \mathrm{~h}^{-1}$ with a single warp and codend, mesh-size $6 \mathrm{~mm}$. A total of 46 trawls (32 in the October 1996 cruise and 14 in May 1998) were conducted. The trawls were carried out between 200 and 1800 m depth. Samples of small suprabenthos/zooplankton were obtained simultaneously during the May 1998 cruise with a small plankton net $(40 \times 60 \mathrm{~cm}$ mouth; $500 \mu \mathrm{m}$ mesh size $)$ attached to the bottom trawl. Also 6 suprabenthonic hauls were completed at $200 \mathrm{~m}$ intervals between 200 and $1800 \mathrm{~m}$. Materials were sorted to the lowest possible taxonomic level and freeze-dried. Fishes and decapod crustaceans were classified to species level, the principal authority for the fishes being Whitehead et al. (1986). Due to the minimum weight required for isotope analysis, suprabenthos/zooplankton was not identified to species level and organisms were grouped into broad taxa. Suprabenthos animals were exclusively caught in the benthic boundary layer ( 1 to $2 \mathrm{~m}$ above bottom), whilst zooplankton (mainly copepods and euphausiids, but also gelatinuous plankton) were caught in this zone and in the water column due to the lack of a closing mechanism on the plankton net. Gelatinous plankton included siphonophores, medusae and chaetognaths, whilst the macrozooplankton was composed of pelagic crustaceans (mainly euphausiids and pelagic decapods). Suprabenthic mysids consisted almost exclusively of the species Boreomysis arctica, and suprabenthic peracarids consisted largely of amphipods, but also of isopods and cumaceans.

White muscle tissue was sampled from each fish, freeze-dried, and ground to a fine homogeneous powder, while most invertebrate samples consisted of whole organisms. The crustacean samples had to be decalcified to remove the inorganic carbon from the exoskeleton, since inorganic carbonates tend to be less negative in $\delta^{13} \mathrm{C}$ than other bodily fractions (DeNiro \& Epstein 1978). A small quantity of $1 \mathrm{M}$ hydrochloric acid was added to the samples and left for $3 \mathrm{~h}$. The samples were then rinsed with distilled water, dried at $60^{\circ} \mathrm{C}$ for $12 \mathrm{~h}$ and reground. Since this process results in enrichment of ${ }^{15} \mathrm{~N}$ (Pinnegar \& Polunin 1999), crustacean samples required analysis before (for $\delta^{15} \mathrm{~N}$ ) and after (for $\delta^{13} \mathrm{C}$ ) decalcification. Some suprabenthos samples could not be analyzed after decalcification due to the small size of the initial sample.

${ }^{15} \mathrm{~N},{ }^{14} \mathrm{~N},{ }^{13} \mathrm{C}$ and ${ }^{12} \mathrm{C}$ concentrations in the samples were determined using continuous-flow isotope-ratio mass spectrometry (CF-IRMS) (Preston \& Owens 1983). One milligram ( $\pm 0.1 \mathrm{mg}$ ) of ground material was oxidized and the $\mathrm{N}_{2}$ and $\mathrm{CO}_{2}$ passed into a single inlet

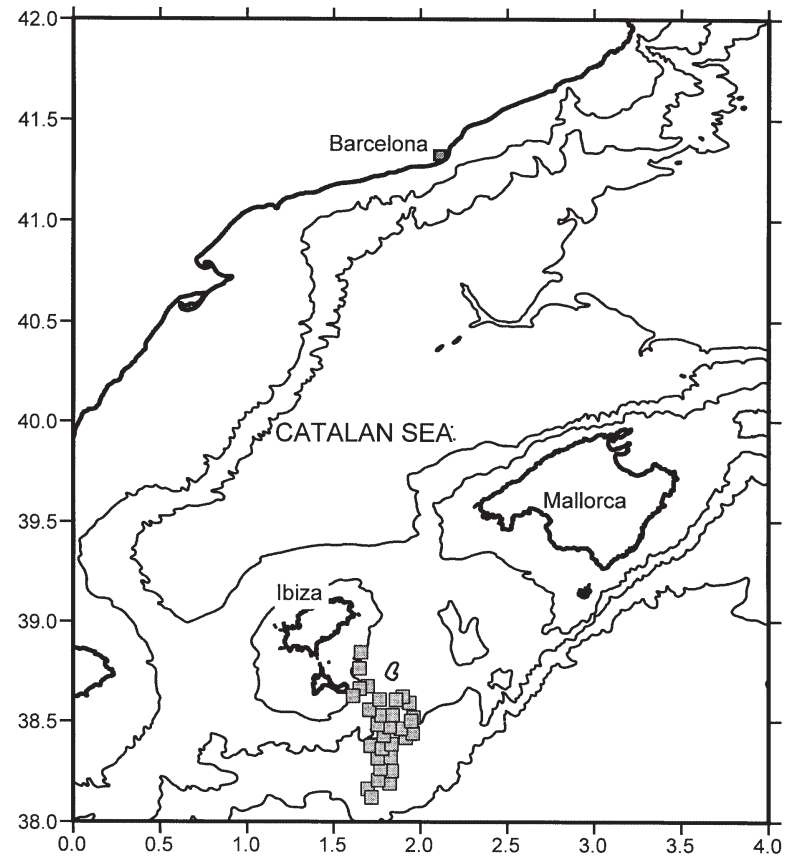

Fig. 1. Location of the study area and specifically of the trawl hauls in October 1996. Depth contours are 200, 1000 and 2000 m. ㄷ: haul sites

dual mass spectrometer (Automated Nitrogen Carbon Analysis [ANCA] SL 20-20 system; PDZ Europa, Crewe, UK). Two samples of an internal reference material (homogenized cod white-muscle tissue) were analyzed after every 6 tissue samples to calibrate the system and compensate for drift with time. Isotope ratios are expressed as parts per thousand (\%) differences from a standard reference material:

$$
\delta X=\left[\frac{\mathrm{R}_{\text {sample }}}{\mathrm{R}_{\text {standard }}}-1\right] \times 10^{3}
$$

where $X$ is ${ }^{15} \mathrm{~N}$ or ${ }^{13} \mathrm{C}, R$ is the ratio of ${ }^{14} \mathrm{~N}:{ }^{15} \mathrm{~N}$ or ${ }^{13} \mathrm{C}:{ }^{12} \mathrm{C}$ for $\mathrm{N}$ or $\mathrm{C}$ isotopes respectively, and $\delta$ is the index of heavy to light isotope in the sample. The standard reference materials were Vienna Pee Dee Belemnite (vPDB) for carbon and atmospheric $\mathrm{N}_{2}$ for nitrogen (Pinnegar \& Polunin 1999). Experimental precision based on the standard deviation of replicates of the internal standard was $0.12 \%$ for $\delta^{15} \mathrm{~N}$ and $0.09 \%$ for $\delta^{13} \mathrm{C}$.

Sample sizes were small ( $<10$ for all samples), but transformation of data was not found to be necessary. Comparisons of $\delta^{15} \mathrm{~N}$ and $\delta^{13} \mathrm{C}$ data were made at species (crustaceans and fishes) and group (suprabenthos and plankton material) levels among the 3 depth zones using 1-way ANOVA ( $\alpha=0.05)$, or Student's $t$-test ( $\alpha=$ 0.05 ) where there was material from only 2 zones; the latter tests were also performed for comparisons between sampling periods. Correlations were used to 
assess the strength of association between $\delta^{15} \mathrm{~N}$ and $\delta^{13} \mathrm{C}$ data across all sampled materials within each depth zone in May 1998; plankton and suprabenthos material were not available for October 1996, and the data from that interval were therefore not correlated. Error terms are expressed as standard deviations (SD) throughout.

\section{RESULTS}

The $\delta^{15} \mathrm{~N}$ of fishes collected during October 1996 ranged from $7.63 \%$ for Alepocephalus rostratus to $13.78 \%$ for the shark Centroscymnus coelolepis (Table 1, Fig. 2), and from $10.08 \%$ for Lampanyctus crocodilus to $13.78 \%$ for Nezumia aequalis during May 1998 (Table 3, Fig. 2). The $\delta^{15} \mathrm{~N}$ of decapod crus- taceans ranged from $7.76 \%$ for the mesopelagic shrimp Sergestes arcticus to $12.43 \%$ for Pontophilus norvegicus in October 1996 (Table 2, Fig. 2), and between $8.78 \%$ for $S$. arcticus and $12.41 \%$ for the polychelid Stereomastis sculpta during May 1998 (Table 4, Fig. 2). The $\delta^{15} \mathrm{~N}$ range exhibited by fishes was wider than that for decapods, and the highest $\delta^{15} \mathrm{~N}$ values were always among the fishes. $\delta^{15} \mathrm{~N}$ values for the suprabenthos/zooplankton collected in May 1998 ranged between $5.25 \%$ (gelatinous plankton) and $8.46 \%$ (macrozooplankton) (Table 5, Fig. 2). These groups of organisms represent a major food source for large fishes and decapods, and consequently the $\delta^{15} \mathrm{~N}$ values were generally below those of decapods (Tables $2 \& 4$ ) and fishes (Tables $1 \& 3$ ).

$\delta^{13} \mathrm{C}$ in fishes ranged from $-19.31 \%$ for the small mesopelagic Cyclothone braueri to $-16.83 \%$ for Con-

Table 1. $\delta^{15} \mathrm{~N}$ and $\delta^{13} \mathrm{C}$ values (mean $\pm \mathrm{SD}$ where appropriate) for fishes from the upper, middle and lower slope zones during October 1996. Key abbreviations are used in Fig. 2

\begin{tabular}{|c|c|c|c|c|c|c|}
\hline Family & Species & Key & Zone & $\delta^{15} \mathrm{~N}(\%)$ & $\delta^{13} \mathrm{C}(\%)$ & (n) \\
\hline \multirow[t]{3}{*}{ Scyliorhinidae } & Galeus melastomus & GM & Lower & $12.28-12.35$ & $-17.34-17.14$ & $(2)$ \\
\hline & Galeus melastomus & GM & Middle & $10.34-10.50$ & $-18.00-17.46$ & (2) \\
\hline & Scyliorhinus canicula & $\mathrm{SC}$ & Upper & $12.33-12.37$ & $-17.40-17.23$ & (2) \\
\hline \multirow[t]{3}{*}{ Squalidae } & Centroscymnus coelolepis & $\mathrm{CC}$ & Lower & $13.78 \pm 0.48$ & $-16.83 \pm 0.09$ & (3) \\
\hline & Dalatias licha & DL & Middle & 13.08 & -16.92 & (1) \\
\hline & Etmopterus spinax & ES & Middle & $12.56-12.96$ & $-17.19-16.80$ & (2) \\
\hline Alepocephalidae & Alepocephalus rostratus & $\mathrm{AR}$ & Lower & $12.04-12.84$ & $-17.96-16.80$ & $(2)$ \\
\hline Gonostomidae & Cyclothone braueri & $\mathrm{CB}$ & Lower & 7.63 & -19.31 & (1) \\
\hline \multirow[t]{2}{*}{ Chauliodontidae } & Chauliodus sloani & $\mathrm{CS}$ & Lower & 10.69 & -18.38 & (1) \\
\hline & Chauliodus sloani & $\mathrm{CS}$ & Middle & 9.94 & -18.58 & (1) \\
\hline Stomiidae & Stomias boa & $\mathrm{SB}$ & Lower & 11.29 & -18.88 & (1) \\
\hline Argentinidae & Argentina sphyraena & AS & Upper & 9.69 & -19.28 & (1) \\
\hline Chlorophthalmidae & Bathypterois mediterraneus & BM & Lower & $12.90 \pm 0.32$ & $-17.45 \pm 0.09$ & $(4)$ \\
\hline Myctophidae & Lampayctus crocodilus & LC & Middle & $9.74-10.05$ & $-18.83-18.52$ & (2) \\
\hline Nettastomidae & Nettastoma melanurum & NM & Middle & 12.35 & -17.16 & (1) \\
\hline \multirow[t]{2}{*}{ Congridae } & Conger conger & $\mathrm{CO}$ & Middle & $13.63-13.71$ & $-16.83-16.81$ & $(2)$ \\
\hline & Conger conger & $\mathrm{CO}$ & Upper & 12.51 & -17.33 & (1) \\
\hline Notacanthidae & Notacanthus bonapartei & NB & Upper & 11.49 & -17.05 & (1) \\
\hline Macroramphosidae & Macroramphosus scolopax & MS & Upper & $12.39-12.89$ & $-19.35-18.07$ & $(2)$ \\
\hline \multirow[t]{5}{*}{ Macrouridae } & Chalinura mediterranea & $\mathrm{CM}$ & Lower & $11.31 \pm 1.11$ & $-17.96 \pm 0.41$ & $(4)$ \\
\hline & Coelorhynchus coelorhynchus & $\mathrm{CC}$ & Upper & $11.17-11.50$ & $-17.48-17.32$ & $(2)$ \\
\hline & Coelorhynchus labiatus & $\mathrm{CL}$ & Lower & $12.40 \pm 0.36$ & $-17.66 \pm 1.44$ & $(4)$ \\
\hline & Nezumia aequalis & NA & Middle & 14.18 & -16.57 & (1) \\
\hline & Trachyrhinchus trachyrhinchus & TT & Middle & $12.84-13.07$ & $-17.60-17.38$ & $(2)$ \\
\hline \multirow[t]{2}{*}{ Merluccidae } & Merluccius merluccius & MM & Middle & 13.92 & -16.73 & (1) \\
\hline & Merluccius merluccius & MM & Upper & $9.96 \pm 0.21$ & $-18.87 \pm 0.13$ & (3) \\
\hline \multirow[t]{3}{*}{ Gadidae } & Gadiculus argenteus & GA & Upper & $9.14-9.83$ & $-18.92-18.72$ & $(2)$ \\
\hline & Micromesistius poutassou & MP & Upper & $10.66-10.67$ & $-18.38-18.16$ & $(2)$ \\
\hline & Phycis blennoides & PB & Upper & $12.30 \pm 0.26$ & $-18.03 \pm 0.28$ & $(4)$ \\
\hline \multirow[t]{3}{*}{ Moridae } & Lepidion lepidion & LL & Lower & $13.42 \pm 0.28$ & $-16.97 \pm 0.20$ & (3) \\
\hline & Lepidion lepidion & LL & Middle & $11.27-11.29$ & $-18.40-17.94$ & $(2)$ \\
\hline & Mora moro & $\mathrm{MO}$ & Middle & $12.78-12.88$ & $-17.07-16.91$ & $(2)$ \\
\hline Apogonidae & Epigonus denticulatus & $\mathrm{ED}$ & Upper & 10.33 & -18.55 & (1) \\
\hline Trichiuridae & Lepidopus caudatus & LE & Upper & 9.74 & -19.13 & (1) \\
\hline Trachichthyidae & Hoplostethus mediterraneus & $\mathrm{HM}$ & Middle & $12.96-14.03$ & $-17.88-17.27$ & $(2)$ \\
\hline Scorpaenidae & Helicolenus dactylopterus & $\mathrm{HD}$ & Upper & $11.76 \pm 0.38$ & $-17.82 \pm 0.28$ & (3) \\
\hline Peristeidae & Peristedion cataphractum & $\mathrm{PC}$ & Upper & $10.46-12.21$ & $-18.43-18.05$ & $(2)$ \\
\hline Scophthalmidae & Lepidorhombus boscii & LB & Upper & $9.30 \pm 0.63$ & $-18.46 \pm 0.49$ & $(4)$ \\
\hline
\end{tabular}


ger conger and Centroscymnus coelolepis during October 1996 (Table 1, Fig. 2), and from $-19.57 \%$ for the epipelagic Trachurus trachurus to $-16.52 \%$ for the macrourid Coelorhynchus coelorhynchus in May 1998 (Table 3, Fig. 2). The $\delta^{13} \mathrm{C}$ in decapod crustaceans spanned from $-20.20 \%$ for the mesopelagic shrimp Sergestes arcticus to $-16.11 \%$ for Pontophilus norvegicus during October 1996 (Table 2), and from $-19.64 \%$ for the crab Geryon longipes to $-17.06 \%$ for the polychelid Stereomastis sculpta during May 1998 (Table 4). The $\delta^{13} \mathrm{C}$ values of suprabenthos and zooplankton ranged from $-22.55 \%$ (calanoid copepods) to $-18.97 \%$ (gelatinous plankton: Table 5). The $\delta^{13} \mathrm{C}$ values of plankton feeders were generally higher (less negative) than those for benthic feeders among both fishes and decapod crustaceans (the latter with the exception of Geryon longipes).

\section{Comparisons between depth zones and sampling periods}

Comparisons among depth zones were possible for 11 species of fishes and 9 species of crustaceans. Among these species, there were no significant depth differences in $\delta^{15} \mathrm{~N}$ for any of the fishes, and only 2 significant differences for the decapod crustaceans; these were for Aristeus anntenatus between the US and LS $\left(\delta^{15} \mathrm{~N}\right.$ of US > LS $t=4.6, \mathrm{p}=0.044)$ and Acanthephyra eximia between the MS and LS $\left(\delta^{15} \mathrm{~N}\right.$ of MS $\left.>\mathrm{LS} ; t=8.92, \mathrm{p}=0.012\right)($ Table 4$)$. For the calanoid copepods $(t=80.83, \mathrm{p}=0.008), \delta^{15} \mathrm{~N}$ was significantly lower in the MS than in the LS. Gelatinous plankton and peracarids differed among all 3 zones (ANOVA, $F=15.16, \mathrm{p}=0.027$ and $F=108.59, \mathrm{p}=0.002$, respectively), both being significantly higher in $\delta^{15} \mathrm{~N}$ at greater depths (US $<$ MS/LS $[t=33.71, \mathrm{p}=0.019$ ] in gelatinous plankton; US/MS $<$ LS $[t=21.76, \mathrm{p}=$ 0.029 ] in peracarids) (Table 5). There were few significant differences in $\delta^{13} \mathrm{C}$ among depth zones, $\delta^{13} \mathrm{C}$ being greater at depth in the fishes Alepocephalus rostratus (US $<\mathrm{MS}, t=7.11, \mathrm{p}=0.019$ ) and Coelorhynchus coelorhynchus (US $<\mathrm{LS}, t=15.05, \mathrm{p}=$ 0.004 )(Table 3). No decapod crustacean species differed significantly in $\delta^{13} \mathrm{C}$ between depth zones
(Tables $2 \& 4$ ), while $\delta^{13} \mathrm{C}$ tended to increase (become less negative) at depth in gelatinous plankton (ANOVA, $F=53.24, \mathrm{p}=0.005$, US $<\mathrm{MS} / \mathrm{LS}$ ) and in peracarids (ANOVA, $F=14.60, p=0.028$; US $>\mathrm{MS} / \mathrm{LS}$ ) (Table 5). There were no significant depth-related differences in macrozooplankton or suprabenthic mysids in either $\delta^{15} \mathrm{~N}$ or $\delta^{13} \mathrm{C}$.

Five comparisons were feasible $(\mathrm{n} \geq 3)$ in fish species within zones between time periods, and 10 in crustacean species, but there were differences between sampling intervals for only 2 crustaceans: in the LS, mean $\delta^{15} \mathrm{~N}$ was greater in May 1998 than in October 1996 in the shrimps Aristeus antennatus and Nematocarcinus exilis, neither of the $\delta^{13} \mathrm{C}$ differences being significant (Tables $2 \& 4$ ). 
Table 2. $\delta^{15} \mathrm{~N}$ and $\delta^{13} \mathrm{C}$ values (mean $\pm \mathrm{SD}$ where appropriate) for crustaceans from the upper, middle and lower slope zones during October 1996. Key abbreviations are used in Fig. 2

\begin{tabular}{|c|c|c|c|c|c|c|}
\hline Family & Species & Key & Zone & $\delta^{15} \mathrm{~N}(\%)$ & $\delta^{13} \mathrm{C}(\%)$ & (n) \\
\hline \multirow[t]{2}{*}{ Aristeidae } & Aristeus antennatus & AA & Upper & $10.96 \pm 0.13$ & $-17.59 \pm 0.18$ & (3) \\
\hline & Aristeus antennatus & AA & Lower & $10.91 \pm 0.28$ & $-17.20 \pm 0.27$ & (6) \\
\hline Crangonidae & Pontophilus norvegicus & $\mathrm{PO}$ & Middle & $12.43 \pm 0.16$ & $-16.11 \pm 0.31$ & (3) \\
\hline \multirow[t]{5}{*}{ Pandalidae } & Plesionika acanthonotus & PA & Middle & $10.52 \pm 0.64$ & $-17.93 \pm 0.45$ & (3) \\
\hline & Plesionika antigai & $\mathrm{PN}$ & Upper & $9.06 \pm 0.14$ & $-18.37 \pm 0.10$ & (3) \\
\hline & Plesionika gigliolii & PG & Upper & $8.56 \pm 0.04$ & $-18.69 \pm 0.21$ & (3) \\
\hline & Plesionika heterocarpus & $\mathrm{PH}$ & Upper & $9.76 \pm 0.13$ & $-18.17 \pm 0.15$ & (3) \\
\hline & Plesionika martia & PR & Upper & $9.51-9.70$ & $-17.95-17.75$ & (2) \\
\hline \multirow[t]{3}{*}{ Pasiphaeidae } & Pasiphaea multidentata & PM & Upper & 9.18 & -18.99 & (1) \\
\hline & Pasiphaea multidentata & $\mathrm{PM}$ & Middle & $9.52-9.64$ & $-19.21-18.63$ & (2) \\
\hline & Pasiphaea sivado & PS & Upper & $9.13 \pm 0.14$ & $-18.87 \pm 0.09$ & (3) \\
\hline \multirow[t]{2}{*}{ Nematocarcinidae } & Nematocarcinus exilis & $\mathrm{NE}$ & Lower & $9.40-9.71$ & $-18.45-18.40$ & (2) \\
\hline & Nematocarcinus sp. & $\mathrm{NE}$ & Lower & $9.92 \pm 0.10$ & $-17.64 \pm 0.28$ & (3) \\
\hline \multirow[t]{3}{*}{ Oplophoridae } & Acanthephyra pelagica & $\mathrm{AP}$ & Middle & $8.33-8.82$ & $-19.01-18.29$ & (2) \\
\hline & Acanthephyra pelagica & $\mathrm{AP}$ & Lower & $8.16 \pm 0.26$ & $-18.74 \pm 0.28$ & (3) \\
\hline & Acanthephyra eximia & $\mathrm{AE}$ & Lower & $10.57 \pm 0.39$ & $-17.82 \pm 0.50$ & (6) \\
\hline Penaeidae & Parapenaeus longirostris & PL & Upper & $9.92 \pm 0.19$ & $-17.99 \pm 0.17$ & (3) \\
\hline \multirow[t]{2}{*}{ Sergestidae } & Sergestes arcticus & SA & Middle & $7.76 \pm 0.53$ & $-20.20 \pm 0.41$ & (3) \\
\hline & Sergia robusta & SR & Lower & $8.02 \pm 0.11$ & $-19.51 \pm 0.92$ & (3) \\
\hline Processidae & Processa caniculata & $\mathrm{PC}$ & Upper & $10.69-11.11$ & $-17.64-17.43$ & (2) \\
\hline Solenoceridae & Solenocera membranacea & SM & Upper & $10.41 \pm 0.10$ & $-17.78 \pm 0.17$ & (3) \\
\hline \multirow[t]{3}{*}{ Galatheidae } & Munida tenuimana & MT & Upper & $9.62-9.99$ & $-18.26-17.86$ & (2) \\
\hline & Munida tenuimana & MT & Lower & $9.78 \pm 0.84$ & $-18.34 \pm 0.24$ & (3) \\
\hline & Munida iris & MI & Upper & $8.50 \pm 0.17$ & $-18.12 \pm 0.30$ & (3) \\
\hline Nephropidae & Nephrops norvegicus & NN & Upper & $9.25 \pm 1.31$ & $-18.27 \pm 0.47$ & (3) \\
\hline Polychelidae & Polycheles typhlops & PT & Middle & $10.78 \pm 0.19$ & $-17.27 \pm 0.25$ & (3) \\
\hline Geryonidae & Geryon longipes & GL & Middle & $11.28 \pm 0.07$ & $-17.17 \pm 0.21$ & (3) \\
\hline Portunidae & Macropipus tuberculatus & MA & Upper & $10.97 \pm 0.48$ & $-18.20 \pm 0.36$ & (3) \\
\hline
\end{tabular}

\section{Relationships between $\delta^{15} \mathrm{~N}$ and $\delta^{13} \mathrm{C}$ data in food webs}

Ascending the food web, from the suprabenthos/zooplankton via decapod crustaceans to the macrourids and sharks, there was a general increase in the $\delta^{15} \mathrm{~N}$ and $\delta^{13} \mathrm{C}$ of consumers (Fig. 2). The correlations between $\delta^{15} \mathrm{~N}$ and $\delta^{13} \mathrm{C}$ of all groups in May 1998 were highly significant in all depth zones (US $\mathrm{r}=0.89$, MS r = 0.82, LS r =0.87; all $\mathrm{p}<0.0001$ ).

\section{DISCUSSION}

Like gut-content data, stable isotopes have several limitations in the extent to which they can elucidate the complex dynamisms of food webs. Among these is the resolution of only broad categories of dietary organisms, distinguishable in terms of $\delta^{15} \mathrm{~N}$ and/or $\delta^{13} \mathrm{C}$ values (Polunin \& Pinnegar in press). The high lipid content of many deep-sea animals may represent another problem, because lipid synthesis favours the lighter isotope ${ }^{12} \mathrm{C}$ over ${ }^{13} \mathrm{C}$ (DeNiro \& Epstein 1977), such that any material which is rich in lipids will be relatively depleted in ${ }^{13} \mathrm{C}$, potentially reducing the chances of correctly identifying types of source material (Pinnegar \& Polunin 1999). Some workers have specifically chosen to sample tissue lipids, since these generally have a fast turnover and integrate short-term dietary histories, but with the high lipid content found among deep-sea animals, we chose to focus on tissues with low levels of lipid wherever possible (e.g. the white muscle of fish). Despite such constraints, stable isotopes have helped here to elucidate ecological features which would otherwise have been very difficult to assess, given the paucity of available dietary data for many deep-sea species.

\section{Temporal changes}

Only 2 significant differences in stable isotope data between the October 1996 and May 1998 intervals were noted, and both of these were increases in $\delta^{15} \mathrm{~N}$ in the latter. Seasonal variations in the $\delta^{15} \mathrm{~N}$ and $\delta^{13} \mathrm{C}$ of planktonic material occur widely (e.g. Wainwright \& Fry 1994), including the Mediterranean (Dauby et al. 1990). $\delta^{15} \mathrm{~N}$ values of surface plankton collected off Corsica were found to be much higher in the spring than in the autumn, since turbulent mixing and 
Table 3. $\delta^{15} \mathrm{~N}$ and $\delta^{13} \mathrm{C}$ values (mean $\pm \mathrm{SD}$ where appropriate) for fishes from the upper, middle and lower slope zones during May 1998. Key abbreviations are used in Fig. 2

\begin{tabular}{|c|c|c|c|c|c|c|}
\hline Family & Species & Key & Zone & $\delta^{15} \mathrm{~N}(\%)$ & $\delta^{13} \mathrm{C}(\%)$ & (n) \\
\hline \multirow[t]{3}{*}{ Scyliorinidae } & Galeus melastomus & GM & Middle & $11.84 \pm 0.42$ & $-17.20 \pm 0.15$ & (3) \\
\hline & Galeus melastomus & GM & Upper & $12.13 \pm 0.38$ & $-17.10 \pm 0.36$ & (3) \\
\hline & Scyliorhinus canicula & $\mathrm{SC}$ & Upper & $11.52 \pm 0.15$ & $-17.74 \pm 0.03$ & (3) \\
\hline \multirow[t]{2}{*}{ Alepocephalidae } & Alepocephalus rostratus & $\mathrm{AR}$ & Middle & $12.07 \pm 0.51$ & $-17.91 \pm 0.19$ & (3) \\
\hline & Alepocephalus rostratus & AR & Upper & $11.35 \pm 0.46$ & $-18.65 \pm 0.04$ & (3) \\
\hline Stomidae & Stomias boa & $\mathrm{SB}$ & Upper & 10.36 & -19.21 & (1) \\
\hline Chlorophthalmidae & Bathypterois mediterraneus & BM & Lower & $13.28 \pm 0.16$ & $-17.48 \pm 0.04$ & (3) \\
\hline \multirow[t]{2}{*}{ Myctophidae } & Lampanyctus crocodilus & $\mathrm{LC}$ & Middle & $10.76 \pm 0.66$ & $-18.27 \pm 0.80$ & (3) \\
\hline & Lampanyctus crocodilus & $\mathrm{LC}$ & Upper & $10.08 \pm 0.44$ & $-18.73 \pm 0.31$ & (3) \\
\hline Congridae & Conger conger & $\mathrm{CO}$ & Middle & 13.26 & -17.78 & (1) \\
\hline \multirow[t]{2}{*}{ Notacanthidae } & Notacanthus bonapartei & NB & Middle & $10.89 \pm 0.11$ & $-16.53 \pm 0.40$ & (3) \\
\hline & Notacanthus bonapartei & NB & Upper & $11.65 \pm 0.05$ & $-17.70 \pm 0.41$ & (5) \\
\hline \multirow[t]{7}{*}{ Macrouridae } & Chalinura mediterranea & $\mathrm{CM}$ & Lower & $12.57 \pm 0.03$ & $-17.52 \pm 0.06$ & (3) \\
\hline & Coelorhynchus coelorhynchus & $\mathrm{CC}$ & Lower & $13.05 \pm 0.49$ & $-16.52 \pm 0.11$ & (3) \\
\hline & Coelorhynchus coelorhynchus & $\mathrm{CC}$ & Upper & $12.14 \pm 0.33$ & $-17.32 \pm 0.03$ & (3) \\
\hline & Nezumia aequalis & NA & Upper & $13.78 \pm 0.61$ & $-17.02 \pm 0.34$ & (6) \\
\hline & Trachyrincus trachyrincus & TT & Middle & 13.36 & -17.14 & (1) \\
\hline & Hymenocephalus italicus & $\mathrm{HI}$ & Middle & $11.31 \pm 0.06$ & $-17.80 \pm 0.02$ & (3) \\
\hline & Hymenocephalus italicus & $\mathrm{HI}$ & Upper & $10.82 \pm 0.46$ & $-18.21 \pm 0.15$ & (3) \\
\hline Merluccidae & Merluccius merluccius & $\mathrm{MM}$ & Upper & $9.86-11.85$ & $-19.11-17.62$ & (2) \\
\hline \multirow[t]{3}{*}{ Gadidae } & Gadiculus argenteus & GA & Upper & $10.32 \pm 0.46$ & $-18.86 \pm 0.11$ & (3) \\
\hline & Micromesistius poutassou & MP & Upper & $10.99 \pm 0.18$ & $-17.94 \pm 0.25$ & (3) \\
\hline & Phycis blennoides & PB & Upper & $12.74 \pm 0.15$ & $-17.79 \pm 0.44$ & (6) \\
\hline \multirow[t]{2}{*}{ Moridae } & Lepidion lepidion & LL & Lower & $13.17 \pm 0.29$ & $-16.97 \pm 0.21$ & (3) \\
\hline & Mora moro & $\mathrm{MO}$ & Upper & $12.69 \pm 0.42$ & $-17.15 \pm 0.19$ & (3) \\
\hline Scorpaenidae & Helicolenus dactylopterus & $\mathrm{HD}$ & Upper & $11.00 \pm 0.33$ & $-18.21 \pm 0.25$ & (3) \\
\hline Scophthalmidae & Lepidorhombus boscii & LB & Upper & 11.07 & -17.48 & (1) \\
\hline \multirow[t]{2}{*}{ Cynoglossidae } & Symphurus ligulatus & SL & Middle & $12.33 \pm 0.41$ & $-17.54 \pm 0.27$ & (3) \\
\hline & Symphurus ligulatus & $\mathrm{SL}$ & Upper & $12.28 \pm 0.08$ & $-17.67 \pm 0.05$ & (3) \\
\hline Carangidae & Trachurus trachurus & TR & Upper & $10.58-11.02$ & $-20.62-18.52$ & (2) \\
\hline
\end{tabular}

Table 4. $\delta^{15} \mathrm{~N}$ and $\delta^{13} \mathrm{C}$ values (mean $\pm \mathrm{SD}$ where appropriate) for crustaceans from the upper, middle and lower slope zones during May 1998. Key abbreviations are used in Fig. 2

\begin{tabular}{|c|c|c|c|c|c|c|}
\hline Family & Species & Key & Zone & $\delta^{15} \mathrm{~N}(\%)$ & $\delta^{13} \mathrm{C}(\%)$ & (n) \\
\hline \multirow[t]{3}{*}{ Aristeidae } & Aristeus antennatus & $\mathrm{AA}$ & Middle & $11.27 \pm 0.33$ & $-17.68 \pm 0.42$ & (14) \\
\hline & Aristeus antennatus & $\mathrm{AA}$ & Lower & $11.34 \pm 0.17$ & $-17.52 \pm 0.32$ & (9) \\
\hline & Aristaeomorpha foliacea & $\mathrm{AF}$ & Upper & $10.47-10.69$ & $-18.97-18.57$ & (2) \\
\hline \multirow[t]{5}{*}{ Pandalidae } & Plesionika acanthonotus & PA & Middle & $10.53 \pm 0.38$ & $-17.76 \pm 0.82$ & (5) \\
\hline & Plesionika edwardsi & $\mathrm{PE}$ & Upper & $9.67 \pm 0.11$ & $-18.90 \pm 0.25$ & (6) \\
\hline & Plesionika gigliolii & PG & Upper & $9.97 \pm 0.17$ & $-18.21 \pm 0.27$ & (4) \\
\hline & Plesionika heterocarpus & $\mathrm{PH}$ & Upper & $10.04 \pm 0.30$ & $-18.08 \pm 0.70$ & (3) \\
\hline & Plesionika martia & PR & Middle & $9.67 \pm 0.18$ & $-18.48 \pm 0.44$ & (3) \\
\hline \multirow[t]{3}{*}{ Pasiphaeidae } & Pasiphaea multidentata & PM & Middle & 9.16 & -18.33 & (1) \\
\hline & Pasiphaea multidentata & PM & Lower & $9.93-10.02$ & $-18.95-18.73$ & (2) \\
\hline & Pasiphaea sivado & PS & Upper & $10.29 \pm 0.09$ & $-19.10 \pm 0.19$ & (4) \\
\hline Nematocarcinidae & Nematocarcinus exilis & $\mathrm{NE}$ & Lower & $10.03-10.61$ & $-17.75-17.53$ & (2) \\
\hline \multirow[t]{3}{*}{ Oplophoridae } & Acanthephyra pelagica & $\mathrm{AP}$ & Lower & $9.07 \pm 0.49$ & $-18.34 \pm 0.21$ & (6) \\
\hline & Acanthephyra eximia & $\mathrm{AE}$ & Middle & $10.56 \pm 0.46$ & $-18.00 \pm 0.34$ & (8) \\
\hline & Acanthephyra eximia & $\mathrm{AE}$ & Lower & $10.62 \pm 0.47$ & $-17.91 \pm 0.49$ & (9) \\
\hline Penaeidae & Parapenaeus longirostris & PL & Upper & $10.02 \pm 0.20$ & $-18.21 \pm 0.34$ & (6) \\
\hline \multirow[t]{3}{*}{ Sergestidae } & Sergestes arcticus & SA & Upper & $8.67-8.89$ & $-20.08-20.52$ & (2) \\
\hline & Sergia robusta & $\mathrm{SR}$ & Middle & $9.27 \pm 0.50$ & $-18.95 \pm 0.61$ & (4) \\
\hline & Sergia robusta & SR & Lower & $8.89 \pm 0.38$ & $-19.32 \pm 0.47$ & (3) \\
\hline Galatheidae & Munida tenuimana & MT & Lower & $10.25 \pm 0.12$ & $-18.25 \pm 0.06$ & (3) \\
\hline \multirow[t]{3}{*}{ Polychelidae } & Polycheles typhlops & $\mathrm{PT}$ & Middle & 10.77 & -17.58 & (1) \\
\hline & Polycheles typhlops & PT & Lower & 12.03 & -16.97 & (1) \\
\hline & Stereomastis sculpta & SS & Lower & 12.41 & -17.06 & (1) \\
\hline Geryonidae & Geryon longipes & GL & Middle & 10.86 & -19.64 & (1) \\
\hline
\end{tabular}


Table 5. $\delta^{15} \mathrm{~N}$ and $\delta^{13} \mathrm{C}$ valuess (mean \pm SD where appropriate) for suprabenthos from the upper, middle and lower slope zones during May 1998. Key abbreviations are used in Fig. 2

\begin{tabular}{|lccccc|}
\hline Family & Key & Zone & $\delta^{15} \mathrm{~N}(\%)$ & $\delta^{13} \mathrm{C}(\%)$ & $(\mathrm{n})$ \\
\hline Gelatinous plankton & $\mathrm{Gp}$ & Upper & $5.03-5.46$ & $-21.63-21.32$ & $(2)$ \\
Gelatinous plankton & $\mathrm{Gp}$ & Middle & $6.90 \pm 0.53$ & $-18.97 \pm 0.92$ & $(4)$ \\
Gelatinous plankton & $\mathrm{Gp}$ & Lower & $6.92 \pm 0.38$ & $-18.67 \pm 0.94$ & $(4)$ \\
Calanoid copepods & $\mathrm{Ca}$ & Middle & $5.47-5.59$ & $-22.77-22.33$ & $(2)$ \\
Calanoid copepods & $\mathrm{Ca}$ & Lower & $6.57 \pm 0.32$ & $-22.04 \pm 0.21$ & $(4)$ \\
Euphausiids & $\mathrm{Eu}$ & Lower & $7.91 \pm 0.31$ & $-19.70 \pm 0.17$ & $(3)$ \\
Macrozooplankton & $\mathrm{Mz}$ & Upper & $8.36 \pm 0.25$ & $-20.67 \pm 0.40$ & $(7)$ \\
Macrozooplankton & $\mathrm{Mz}$ & Middle & $8.46 \pm 0.35$ & $-20.70 \pm 0.71$ & $(4)$ \\
Macrozooplankton & $\mathrm{Mz}$ & Lower & $8.21 \pm 0.60$ & $-20.51 \pm 0.33$ & $(8)$ \\
Suprabenthic mysids & $\mathrm{Sm}$ & Middle & $8.16 \pm 1.05$ & $-20.23 \pm 0.56$ & $(3)$ \\
Suprabenthic mysids & $\mathrm{Sm}$ & Lower & 8.49 & -20.21 & $(1)$ \\
Suprabenthic peracarids & $\mathrm{Sp}$ & Upper & $7.26-7.39$ & $-21.59-21.38$ & $(2)$ \\
Suprabenthic peracarids & $\mathrm{Sp}$ & Middle & $7.39-7.59$ & $-21.18-20.33$ & $(2)$ \\
Suprabenthic peracarids & $\mathrm{Sp}$ & Lower & $8.41 \pm 0.34$ & $-20.01 \pm 0.78$ & $(3)$ \\
\hline
\end{tabular}

both cases, feeding at lower trophic levels at greater depth is indicated, and this was corroborated by the $\delta^{15} \mathrm{~N}$ data. In contrast, calanoid copepods (1 to $3.5 \mathrm{~mm}$ ), peracarids ( 2 to $20 \mathrm{~mm}$ ) and the gelatinous plankton (4 to $20 \mathrm{~mm}$ ), all exhibited significantly higher $\delta^{15} \mathrm{~N}$ values at greater depth; there are 2 hypotheses as to why this might be so. One hypothesis is that the consumers of the suprabenthos and zooplankton have on average more trophic steps between them and the basal materials at depth, while the other is that the food-web basal materials become ${ }^{15} \mathrm{~N}$-enriched at depth.

Longer food chains leading to the planktonic animals are another possible explanation for the greater $\delta^{15} \mathrm{~N}$ of

upwelling caused by offshore winds bring nitrate which is high in $\delta^{15} \mathrm{~N}$ to the surface where it is taken up by phytoplankton (Michener \& Schell 1994). As this mixing and upwelling ceases, the surface waters rapidly become oligotrophic and primary production becomes largely dependent on recycled nitrogen, especially in the form of ammonia which is generally depleted in ${ }^{15} \mathrm{~N}$. Many small invertebrates feed nonselectively on the fallen detritus or plankton, and because they generally posses rapid tissue turnover times, they might be expected to exhibit isotopic compositions which follow these seasonal patterns in the plankton. The shrimps Aristeus antennatus and $\mathrm{Ne}$ matocarcinus exilis did show higher $\delta^{15} \mathrm{~N}$ values in the spring of 1998 than autumn of 1996. However we would not generally expect organisms feeding at higher trophic levels (e.g. fishes and large crustaceans) to express seasonal patterns in isotope composition, because of their much slower rates of tissue turnover (e.g. Hesslein et al. 1993).

\section{Depth changes}

Of the only 2 macroconsumer species showing significant differences in stable isotope data among depth zones, one, Aristeus antennatus, has a diet which varies from primarily benthic invertebrates (mainly polychaetes and other infaunal taxa) and suprabenthic crustaceans in shallow water to a high proportion of less-mobile prey from low trophic levels such as foraminiferans and pteropod-shell remains in deeper regions (Cartes 1994). The other macroconsumer species, Acanthephyra eximia, is generally carnivorous in shallower water, but shifts to a more detritivorous mode of feeding in the deeper zone (Cartes 1993). In these consumers in deeper water. Changes in the dominant feeding type of copepods and other mesozooplakton have been observed with increasing depth, with an increase in carnivorous forms occurring in deeper water (Vinogradov \& Tseitlin 1983). Although the feeding habits of suprabenthos are poorly known, the mysid Boreomysis arctica and the amphipod Rhachotropis caeca, which are dominant suprabenthic species off Ibiza, feed on crustacean remains (particularly copepods), and become more common with increasing water depth (Cartes \& Sorbe 1998).

Saprotrophic fungi and other microbes are known to enrich organic matter in ${ }^{15} \mathrm{~N}$ by preferentially utilizing the labile matter that is relatively abundant in ${ }^{14} \mathrm{~N}$ (Gleixner et al. 1993, Hobbie \& Macko 1999). Refractory substances like the chitin from copepod exoskeletons thus become relatively more abundant in marine snow as it sinks (Miquel et al. 1994). Chitin is often overlooked as an important source of nitrogen in the marine environment (Shimmelmann et al. 1998), and the ability to utilise chitin has been demonstrated for bacteria (Kichner 1995) and many types of crustaceans (Bucholz \& Vetter 1993, Watanabe et al. 1998) which are common consumers in the deep sea. The high and depth-stable temperature in the Mediterranean Sea (Fredj \& Laubier 1985) must facilitate microbial degradation of particulate organic matter in the water column. Bacteria are recognised as having an important role in sustaining the benthos of many deep oligotrophic areas of this sea (Danovaro et al. 1993).

$\delta^{13} \mathrm{C}$ values may be useful indicators of source materials, since fractionation of the carbon isotopes with each trophic level is small relative to that exhibited by nitrogen isotopes, and there are often distinct differences among types of basal materials in ecosystems. In the present study, species depleted in ${ }^{13} \mathrm{C}$ generally fed 
near the base of the food web and were more closely associated with the plankton. The higher $\delta^{13} \mathrm{C}$ values of the fishes Alepocephalus rostratus and Coelorhynchus coelorhynchus at greater depth (Table 3) did not seem to corroborate available gut-content data. The related Coelorhynchus labiatus appears to feed more on suprabenthos and zooplankton in deeper waters (Carrassón 1994). A. rostratus feeds primarily on macroplankton on the lower slope (Carrassón \& Matallanas 1990). These are both diets which might have been expected to produce lower values of $\delta^{13} \mathrm{C}$ at greater depth. In fact, $\delta^{13} \mathrm{C}$ values of the suprabenthos and zooplankton tended also to increase at greater depth and, as for nitrogen, this is consistent with the biodegradation of marine snow leaving residues of more refractory materials (Williams \& Gordon 1970, Mullerniklas et al. 1994) such as inorganic carbonates and chitin, which are known to be enriched in ${ }^{13} \mathrm{C}$ compared to other biochemical fractions (Bunn et al. 1995).

\section{Food-web structure}

The strong correlations observed between $\delta^{15} \mathrm{~N}$ and $\delta^{13} \mathrm{C}$ (Fig. 2) are indicative of a single type of primary source material supporting deep-sea communities. Vertical fluxes of organic particulate matter have been measured in the study region, the rates of organic carbon at 800 to $1000 \mathrm{~m}$ depth being 1 to $5 \mathrm{~g} \mathrm{~m}^{-2} \mathrm{yr}^{-1}$ (Miquel et al. 1994). The correlations between $\delta^{15} \mathrm{~N}$ and $\delta^{13} \mathrm{C}$ contrast with those in communities of the Mediterranean littoral (e.g. cf. Lepoint et al. 2000 and Pinnegar \& Polunin 2000), where an array of possible sources of production including plankton, macroalgae and seagrasses, are available, and the relationship between $\delta^{15} \mathrm{~N}$ and $\delta^{13} \mathrm{C}$ is much weaker (e.g. consumers in Pinnegar \& Polunin 2000, $\mathrm{r}=0.46$; nonepiphytic consumers in Lepoint et al. 2000, $\mathrm{r}=0.24$ ). Being a short distance away from the Balearic Islands, our study area experienced lower vertical flux rates than oceanic areas closer to land (Danovaro et al. 1999), and the sediments have very little input of terrigenous material (Emelyanov 1972). If falls of large epipelagic predators such as sharks or tunas had been significant food sources, the materials apparently supporting the bathyal food webs studied here would have been higher and more variable in $\delta^{15} \mathrm{~N}$ and $\delta^{13} \mathrm{C}$. If macrophytes such as large algae had been significant contributors, then higher and more variable values of $\delta^{13} \mathrm{C}$ would have been indicated for the source materials of the deep-sea food webs than were found. Marine snow almost certainly constitutes the main supply of food materials to the sea floor in the study area. However, values for the slope of regression between $\delta^{15} \mathrm{~N}$ and $\delta^{13} \mathrm{C}$ of consumers differed from the value would be expected (a slope of $\sim 3$ ) if $\delta^{15} \mathrm{~N}$ increased by 3 to $3.4 \%$ and $\delta^{13} \mathrm{C}$ by $1 \%$ (DeNiro \& Epstein 1978) at each trophic level.

The spread of $\delta^{15} \mathrm{~N}$ and $\delta^{13} \mathrm{C}$ values presented here for fishes and decapods is generally consistent with the highly diversified diets of large consumers in the western Mediterranean bathyal zone, as indicated by gut-content data (e.g. Macpherson 1977, Cartes 1998). There appear to be 3 groups of fishes, namely those with a low trophic level, which consume a variety of planktonic foods (copepods, gelatinous plankton, mesopelagic decapods and euphausiids), those which preferentially consume benthic/suprabenthic invertebrates, and the top predators (e.g. sharks, some macrourids and Conger conger) which prey on large decapods, other fishes and cephalopods (e.g. Carassón et al. 1992).

\section{Trophic level}

We estimated the trophic level (TL) of the top predators based on their $\delta^{15} \mathrm{~N}$ data and using calanoid copepods as a reference material (with an assumed trophic level of 2) and an increase in $\delta^{15} \mathrm{~N}$ of $3.4 \%$ per trophic level (Vander Zanden et al. 1997). In this way, the maximum trophic level exhibited by any fish species was 4.4 for both the shark Centroscymnus coelolepsis (October 1996) and the macrourid Nezumia aequalis (May 1998). This is equivalent to the trophic level occupied elsewhere by large sharks (Cortés 1999), and generally agrees with the suggestion that aquatic food chains typically have no more than 4 or 5 trophic steps (Hall \& Raffaelli 1993). Bathyal fishes off the Balearic Islands appeared to be foraging over 2 to 3 full trophic levels, while decapods fed over 2 trophic levels, as did the suprabenthos/zooplankton. Calanoid copepods appeared to feed at a lower trophic level than did the macrozooplankton, euphausiids and suprabenthic peracarid groups. The gelatinous plankton generally exhibited very low $\delta^{15} \mathrm{~N}$ values, and this may indicate a greater reliance on POM or microzooplankton (Malej et al. 1993).

Comparing the results presented here for deep-sea consumers with those from littoral environments (e.g. Lepoint et al. 2000, Pinnegar \& Polunin 2000), zooplankton at shallower depths generally exhibited lower $\delta^{15} \mathrm{~N}$ values $(\sim 3.5 \%$ ) than those of zooplankton from the deep waters ( 5.5 to $6.5 \%$ ), and this was also the case for gelatinous plankton, decapod crustaceans, fishes, mysids and peracarids. This suggests that it is variation in the underlying $\delta^{15} \mathrm{~N}$ of the basal organisms that is responsible for apparent TL differences between littoral and deep-sea organisms, and not any fundamental difference in TL of the large consumers in the food webs concerned. 
Acknowledgements. Analyses were conducted by S. Barker of the Biomedical Mass-Spectrometry Unit, University of Newcastle upon Tyne. J.K.P. was supported by a studentship from the Fisheries Society of the British Isles and N.V.C.P. by a grant from the Foundation for Environmental Conservation. The EU (project FAIR CT-95 0655) funded the cruises, and we are grateful to the participants and crew of those vessels.

\section{LITERATURE CITED}

Andersen V, Sardou J, Nival P (1992) The diel migrations and vertical distrivbutions of zooplankton and micronekton in the northwestern Mediterranean Sea. 2. Siphonophores, hydromedusae and pyrosomids. J Plankton Res 14:1155-1169

Azam F, Fenchel T, Field JG, Gray JS, Meyer-Reil LA, Thingstad $F$ (1983) The ecological role of water-column microbes in the sea. Mar Ecol Prog Ser 10:257-263

Bucholz F, Vetter RAH (1993) Enzyme-kinetics in cold water characteristics of $\mathrm{n}$-acetyl-beta-d-glucosaminidase activity in the Antarctic krill Euphausia superba, compared with other crustacean species. J Comp Physiol B Biochem Syst Environ Physiol 163:28-37

Bunn, SE, Loneragan NR, Kempster MA (1995) Effects of acid washing on stable isotope ratios of $\mathrm{C}$ and $\mathrm{N}$ in peneid shrimp and seagrass: implication for food web studies using multiple stable isotopes. Limnol Oceanogr 40: 622-625

Carassón M (1994) Relaciones tróficas en las comunidades ícticas bentónicas (de 1000 a 2200 m) del Mar Catalán. Doctoral thesis, Universidad Autónoma de Barcelona

Carassón M, Matallanas J (1990) Preliminary data about the feeding habits of some deep-sea Mediterranean fishes. J Fish Biol 36:461-463

Carassón M, Stefanescu C, Cartes JE (1992) Diets and bathymetric distributions of two bathyl sharks of the Catalan deep-sea (western Mediterranean). Mar Ecol Prog Ser 82: 21-30

Cartes JE (1993) Feeding habits of oplophorid shrimps in the deep western Mediterranean. J Mar Biol Assoc UK 73: 193-206

Cartes JE (1994) Influence of depth and season on the diet of the deep-water aristeid Aristeus antennatus along the continental slope (400-2300 m) in the Catalan Sea (western Mediterranean). Mar Biol 120:639-648

Cartes JE (1998) Feeding strategies and partition of food resources in deep-water decapod crustaceans (400-2300 m). J Mar Biol Assoc UK 78:509-524

Cartes JE, Sorbe JC (1998) Aspects of population structure and feeding ecology of the deep-water mysid Boreomysis arctica, a dominant species in western Mediterranean slope assemblages. J Plankton Res 20:2401-2411

Cortés E (1999) Standardized diet compositions and trophic levels of sharks. ICES J Mar Sci 56:707-717

Danovaro R, Fabiano M, Della Croce N (1993) Labile organic matter and microbial biomasses in deep-sea sediments (Eastern Mediterranean Sea). Deep-Sea Res 40:953-965

Danovaro R, Dinet A, Duineveld G, Tselepides A (1999). Benthic response to particulate fluxes in different trophic environments: a comparison between the Gulf of LionsCatalan Sea (western Medieterranean) and the Cretan Sea (eastern Mediterranean). Prog Oceanogr 44:287-312

Dauby P, Mosora F, Vertez M (1990) A yearly study of ${ }^{13} \mathrm{C} /{ }^{12} \mathrm{C}$ isotope variations in the Calvi Bay plankton. Rapp Comm Int Mer Méditer 32:202

DeNiro MJ, Epstein S (1977) Mechanism of carbon isotope fractionation associated with lipid synthesis. Science 197: 261-263

DeNiro MJ, Epstein S (1978) Influence of diet on the contribution of carbon isotopes in animals. Geochim Cosmochim Acta 42:495-506

Emelyanov EM (1972) Principal types of recent bottom sediments in the Mediterranean Sea: their mineralogy and geochemestry. In: Stanley DJ (ed) The Mediterranean Sea: a natural sedimentation laboratory. Dowden, Hutchinson \& Ross, Stroudsburg, p 355-386

Estrada M, Vives F, Alvárez M (1985) Life and the productivity of the open sea. In Margalef R (ed) Key environments: western Mediterranean. Permagon Press, Oxford, p 148-197

Fredj G, Laubier L (1985) The deep Mediterranean benthos. In Moraitou-Apostolopoulou M, Kiortsis V (eds) Mediterranean marine ecosystems. Plenum Press, New York, p 109-146

Gage JD, Tyler PA (1991) Deep-sea Biology: a Natural History of Organisms at the Deep Sea Floor. Cambridge University Press, Cambridge

Gleixner G, Danier H, Werner RA, Schimdt HL (1993) Correlations between the ${ }^{13} \mathrm{C}$ content of primary and secondary plant products in different cell compartments and that in decomposing Basidiomycetes. Plant Physiol (Wash DC) 102:1287-1290

Hall SJ, Raffaelli DG (1993) Food webs: theory and reality. Adv Ecol Res 24:187-239

Harrigan P, Zieman JC, Macko SA (1989) The base of nutritional support for the gray snapper (Lutjanus griseus): an evaluation based on combined stomach content and stable isotope analysis. Bull Mar Sci 44:65-77

Hesslein RH, Hallard KA, Ramal P (1993) Replacement of sulphur, carbon, and nitrogen of growing broad whitefish (Coregonus nasus) in response to a change in diet traced by ${ }^{34} \mathrm{~S},{ }^{13} \mathrm{C}$, and ${ }^{15} \mathrm{~N}$. Can J Fish Aquat Sci 50:2071-2076

Hobbie EA, Macko SA (1999) Insights into nitrogen and carbon dynamic of ectomycorrizal and saprotrophic fungi from isotopic evidence. Oecologia 118:353-360

Kirchner M (1995) Microbial colonisation of copepod body surfaces and chitin degradation in the sea. Helgol Meeresunters 49:201-212

Koslow JA (1996) Energetic and life-history patterns of deepsea benthic, benthopelagic and seamount associated fish. J Fish Biol 49 (Suppl A):54-74

Lepoint G, Nyssen F, Gobert S, Dauby P, Bouquegneau JM (2000) Relative impact of a seagrass bed and its adjacent epilithic algal community in consumer diets. Mar Biol 136: $513-518$

Macpherson E (1977) Estudio sobre relaciones tróficas en peces bentónicos de la costa catalana. Doctoral thesis, Universidad de Barcelona

Macquart-Moulin C (1993) Vertical distribution, surface stratifications and migrations of pelagic mysids and amphipods over the French Atlantic and Mediterranean margins. J Plankton Res 15:1149-1170

Mahaut M, Geistdoerfer P, Sibuet M (1990) Trophic strategies in carnivorous fishes: Their significance in energy transfer in the deep-sea benthic ecosystem (Meriadzek Terrace Bay of Biscay). Prog Oceanogr 24:223-237

Malej A, Faganeli J, Pezdi J (1993) Stable isotope and biochemical fractionation in the marine pelagic food chain: the jellyfish Pelagia noctiluca and net zooplankton. Mar Biol 116:565-570

Maynou F, Cartes JE (2001) Community structure of bathyal decapod crustacean assemblages off the Balearic Islands (south-western Mediterranean): seasonal changes and regional patterns in zonation. J Mar Biol Assoc UK (in press) 
Michener RH, Schell DM (1994) Stable isotope ratios as tracers in marine aquatic food webs. In: Lajtha K, Michener $\mathrm{RH}$ (eds) Stable isotopes in ecology and environmental science. Blackwell Scientific, Oxford, p 138-157

Miquel JC, Fowler SW, La Ros J, Buat-Menard P (1994) Dynamics of the downward flux of particles and carbon in the open north-west Mediterranean. Deep-Sea Res 41: 243-261

Moranta J, Stefanescu C, Massutí E, Morales-Nin B, Lloris D (1998) Fish community structure and depth-related trends on the continental slopes of the Balearic Islands (Algerian basin, western Mediterranean). Mar Ecol Prog Ser 171: $247-259$

Mullerniklas G, Schuster S, Kaltenbock E, Herndl GJ (1994) Organic content and bacterial metabolism in amorphous aggregations of the northern Adriatic Sea. Limnol Oceanogr 39:58-68

Pérès JM (1985) History of the Mediterranean biota and the colonization of the depths. In: Margalef R (ed) Key environments: western Mediterranean. Permagon Press, Oxford, p 198-232

Pinnegar JK, Polunin NVC (1999) Differential fractionation of $\delta^{13} \mathrm{C}$ and $\delta^{15} \mathrm{~N}$ among fish tissues: implications for the study of trophic levels. Funct Ecol 13:225-231

Pinnegar JK, Polunin NVC (2000) Contributions of stable-isotope data to elucidating food webs of Mediterranean rocky littoral fishes. Oecologia 122:399-409

Polunin NVC, Pinnegar JK (in press) Ecology of fishes in marine food-webs. In: Hart PJB, Reynolds JD (eds) Handbook of fish and fisheries. Blackwell, Oxford

Preston T, Owens NJP (1983) Interfacing an automatic elemental analyser with an isotope ratio mass spectrometer: the potential for fully automated total nitrogen and nitrogen-15 analysis. Analyst 108:971-977

Randall DJ, Farrell AP (eds) (1997) Deep-sea fishes. Academic Press, New York

Riemann F (1989) Gelatinous phytoplankton detritus aggregates on the Atlantic deep-sea bed: structure and mode of formation. Mar Biol 100:533-539

Shimmelmann A, Wintsch RP, Lewan MD, DeNiro MJ (1998)

Editorial responsibility: Otto Kinne (Editor),

Oldendorf/Luhe, Germany
Chitin: 'forgotten' source of nitrogen - from modern chitin to thermally mature kerogen, lessons from nitrogen isotope ratios. ACS (Am Chem Soc) Symp Ser 707:226-242

Smith CR, Hoover DJ, Doan SE, Pope RH, Demaster FC, Altabet MA (1996) Phytodetritus at the abyssal seafloor across $10^{\circ}$ of latitude in the central equatorial Pacific. Deep-Sea Res 43:1309-1338

Stefanescu C, Lloris D, Rucabado J (1993) Deep-sea fish assemblages in the Catalan Sea (western Mediterranean) below a depth of 1000 m. Deep-Sea Res 40:695-707

Sumich JL (1999) An introduction to the biology of marine life, 7 th edn. WCB McGraw-Hill, New York

Vander Zanden MJ, Cabana G, Rasmussen JB (1997) Comparing trophic position of freshwater fish calculated using stable nitrogen isotope ratios $\left(\delta^{15} \mathrm{~N}\right)$ and literature dietary data. Can J Fish Aquat Sci 54:1142-1158

Vetter EW, Dayton PK (1999) Organic enrichment by macrophyte detritus, and abundance patterns of megafaunal populations in submarine canyons. Mar Ecol Prog Ser 186: 137-148

Vinogradov ME, Tseitlin VB (1983) Deep-sea pelagic domain (aspects of bioenergetics). In: Rowe GT (ed) Deep-sea biology. The sea, Vol 8. John Wiley, New York, p 123-165

Wainwright SC, Fry B (1994) Seasonal variation of the stable isotope compositions of coastal marine plankton from Woods Hole. Estuaries 17:552-560

Watanabe T, Kono M, Aida K, Nagasawa H (1998) Purification and molecular cloning of a chitinase expressed in the hepatopancreas of the penaeid prawn Penaeus japonicus. Biochim Biophys Acta Prot Struct Mol Enzym 1382: 181-185

Whitehead PJP, Bauchot ML, Hureau JC, Neilsen J, Tortonese E (1986) Fishes of the North-Western Atlantic and the Mediterranean. UNESCO, Paris

Williams PM, Gordon LI (1970) ${ }^{13} \mathrm{C}:{ }^{12} \mathrm{C}$ ratios in dissolved and particulate organic matter in the sea. Deep-Sea Res 17: $19-27$

Witte U (1999) Consumption of large carcasses by scavenger assemblages in the deep Arabian Sea: observations by baited camera. Mar Ecol Prog Ser 183:139-147

Submitted: August 8, 1999; Accepted: February 15, 2001

Proofs received from author(s): September 10, 2001 